NSF-KITP-09-53

SLAC-PUB-13584

SU-ITP-09/16

BRX-TH-607

\title{
Insightful D-branes
}

\author{
Gary Horowitz ${ }^{1}$, Albion Lawrence ${ }^{2,4}$, and Eva Silverstein ${ }^{3,4}$ \\ ${ }^{1}$ Department of Physics, University of California, Santa Barbara, CA 93106 \\ ${ }^{2}$ Brandeis Theory Group, Brandeis University, MS 057, PO Box 549110, Waltham, MA 02454 \\ ${ }^{3}$ SLAC and Department of Physics, Stanford University, Stanford, CA 94305-4060 \\ ${ }^{4}$ Kavli Institute for Theoretical Physics, University of California, Santa Barbara, CA 93106
}

We study a simple model of a black hole in AdS and obtain a holographic description of the region inside the horizon. A key role is played by the dynamics of the scalar fields in the dual gauge theory. This leads to a proposal for a dual description of D-branes falling through the horizon of any AdS black hole. The proposal uses a field-dependent time reparameterization in the field theory. We relate this reparametrization to various gauge invariances of the theory. Finally, we speculate on information loss and the black hole singularity in this context.

April 2009 


\section{Introduction}

A longstanding problem in gauge/gravity duality is to describe the region inside a black hole horizon in terms of the dual gauge theory. There have been various interesting approaches to this problem [1,2,3] but none have directly addressed the question of how to describe the observations of an infalling observer. We will present a proposal for how to do this, using the dynamics of rolling scalar fields in the gauge theory and their manifestation in terms of D-brane probes on the gravity side.

Consider the patch of $A d S_{5}$, with metric

$$
d s^{2}=\frac{r_{p}^{2}}{\ell^{2}}\left(-d t_{p}^{2}+t_{p}^{2} d \sigma^{2}\right)+\frac{\ell^{2}}{r_{p}^{2}} d r_{p}^{2} .
$$

Here we have started with Poincaré coordinates, but replaced the constant- $r_{p}$ Minkowski space slices $\mathbb{R}^{3,1}$ with a patch of Minkowski space consisting of the backward light cone of a point; this patch is described by Milne coordinates with spatial slices equal to hyperbolic 3 -space $\mathbb{H}_{3}$, with constant curvature metric $d \sigma^{2}$. The dual gauge theory describing this lives on the geometry

$$
d s^{2}=-d t_{p}^{2}+t_{p}^{2} d \sigma^{2}
$$

or equivalently on the static cylinder geometry

$$
d s^{2}=-d \eta^{2}+\ell^{2} d \sigma^{2}
$$

obtained from (1.2) by a conformal transformation that is valid away from $t_{p}=0$.

Placing a D3-brane at constant $r_{p}$ is dual to the gauge theory with a scalar field VEV turned on breaking its $U(N)$ gauge symmetry down to $U(N-1) \times U(1)$. The radial collective coordinate of the D3-brane, which corresponds to the VEV of the scalar field eigenvalue, is governed at low energies by the DBI action. This is obtained by integrating out the other degrees of freedom in the gauge theory.

Compactifying the hyperbolic space, by orbifolding $\mathbb{H}_{3}$ by a discrete group of isometries $\Gamma$, leads to a spacetime with a singularity at $t_{p}=0$. As we will review in $\S 2$, this spacetime is in fact a static black hole [4] whose event horizon is shown in figure 1a. (This is a higher dimensional generalization of the BTZ black hole in three dimensions. $)^{2}$

1 A similar action describes the low energy dynamics of the full set of low energy degrees of freedom on the D3-brane. One can also generalize to a stack of $n \ll N$ D3-branes with interacting degrees of freedom; a fair amount is known about this "non-abelian DBI" action.

2 Other singularities in spacetimes with holographic duals have been described in [0],6]. These correspond to gauge theories with dimensionful or time-dependent couplings, so there is no static, non-singular presentation of the gauge theory as our system has. 


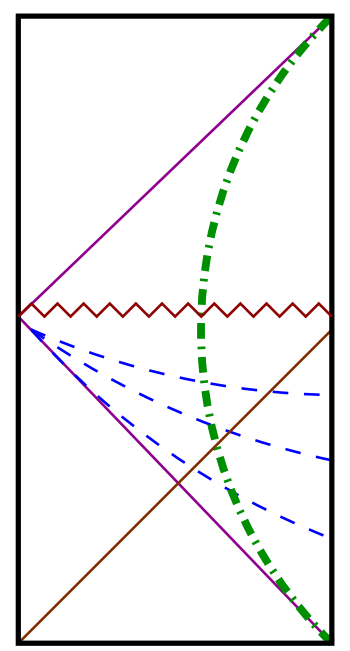

(A)

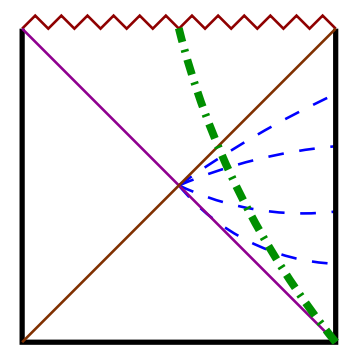

(B)

Fig. 1: A black hole can be obtained from the Poincare patch by compactifying along hyperbolic spatial slices. The (green) dash-dot line denotes a D3-brane probe.

(a) The (blue) dashed lines denote spatial slices in Poincaré coordinates (b) The (blue) dashed lines are spatial slices in Schwarzschild coordinates.

In the figure, we also indicate the trajectory of the D3-brane at constant $r_{p}$. Well before approaching the singularity, while its dynamics is still described to a very good approximation by the DBI action, this D3-brane falls through the horizon of the black hole. An infalling observer on the D3-brane is thus described in the Yang-Mills theory by the low energy effective action for the scalar field $r_{p}$ and its superpartners. We will show that these variables remain classical through the horizon, providing a simple description of an observer falling inside the black hole.

An observer outside the black hole, on the other hand, can be described on the gravity side by standard Schwarzschild coordinates [4], with metric:

$$
d s^{2}=-\left(\frac{r^{2}}{\ell^{2}}-1\right) d t^{2}+\frac{d r^{2}}{\left(\frac{r^{2}}{\ell^{2}}-1\right)}+r^{2} d \sigma^{2}
$$

The equal- $t$ time slices are depicted in figure $1 \mathrm{~b}$. As we will describe below, the field theory duals of the two descriptions are related by a conformal transformation to a static cylinder (1.3) combined with a field-dependent time reparameterization. This second set of gauge theory variables describes the black hole in terms of a thermal field theory. The combined transformation produces a DBI action in Schwarzschild coordinates. This effective action breaks down near the horizon because of the coordinate singularity there. It takes forever 
to reach the horizon in Schwarzschild time $t$, and as we will discuss further below the fluctuations about the classical solution grow large in these variables. These features reflect the thermalization of initial excitations in the gauge theory.

Nonetheless, both sets of variables descend from the Yang-Mills description, as we will describe in $\S 3$, and so one should be able to map phenomena seen in one set of variables to phenomena in another. We will describe and compare the physics in different frames in $\S 3$, and construct the transformation of quantum variables between these frames in $\S 4$. Given these results, we can begin to attack some well-known problems in black hole physics.

The black hole can be formed naturally from a collapsing shell of $N$ D3-branes, and eventually decays as we will discuss in $\S 5$. The description of the black hole interior provided by the scalar field dynamics may help us gain insight into the problem of information loss. Although we began with hyperbolic black holes derived in the Poincaré patch of $A d S_{5}$, our proposal can be generalized to apply to other types of black holes as well.

Having found a way to follow matter falling inside the horizon, one can now ask what happens near the black hole singularity. At present our discussion is necessarily qualitative since both the bulk and the boundary theory become strongly coupled. Nevertheless, an interesting picture emerges, which we also discuss in $\S 5$. The physics near the singularity is associated with a rolling scalar field, just like earlier discussions of singularities in AdS [7,8]. However, there are several crucial differences. In these papers, the bulk singularities only arose after the field theory was modified with a multi-trace operator which produced a potential unbounded from below. The Hamiltonian was ill defined without some prescription for a self adjoint extension, and the singularity was associated with the scalar field rolling off to infinity; that is, with ultraviolet physics. In the present case, although we again have a potential unbounded from below, there is no need for multi-trace operators or self adjoint extensions. Furthermore, in our class of examples, the location of the would-be singularity is at the origin in scalar field space rather than at infinity, a regime governed by infrared physics, specifically the corresponding effects of the extra light states at the origin along the lines of [9, 10,11].3 In particular, near the singularity there is no local description of the physics in terms of scalar field eigenvalues, since the full set of $U(N)$ adjoint matrix degrees of freedom participates in the dynamics.

We will present some further speculations and directions for future work in $\S 6$.

3 See [12] for a recent discussion of these effects in black hole physics in AdS/CFT, and [13] for previous discussions of related effects. 


\section{Hyperbolic black holes}

The metric (1.1) has a Killing field which is timelike near infinity and spacelike near the singularity. To make this manifest, we introduce new coordinates

$$
t_{p}=-\frac{r \ell e^{-t / \ell}}{\left(r^{2}-\ell^{2}\right)^{1 / 2}}, \quad r_{p}=\left(r^{2}-\ell^{2}\right)^{1 / 2} e^{t / \ell}
$$

The metric becomes

$$
d s^{2}=-f(r) d t^{2}+\frac{d r^{2}}{f(r)}+r^{2} d \sigma^{2}
$$

where

$$
f(r)=\frac{r^{2}}{\ell^{2}}-1 .
$$

We can orbifold $\mathbb{H}_{3}$ by a discrete group $\Gamma$, leading to a compact hyperbolic space $\Sigma=$ $\mathbb{H}_{3} / \Gamma$. $d \sigma^{2}$ now denotes the metric on $\Sigma$. The metric (2.2) takes the standard form of a static black hole with horizon at $r=\ell$ having constant negative curvature. The inverse transformation, taking (2.2) into (1.1), is

$$
r=-\frac{r_{p} t_{p}}{\ell}, \quad t=-\frac{\ell}{2} \ln \left(\frac{t_{p}^{2}}{\ell^{2}}-\frac{\ell^{2}}{r_{p}^{2}}\right)
$$

These transformations are valid outside the horizon, i.e., $r>\ell$. Analogous formula apply for $r<\ell$. However, a key point for our later discussion is that the Poincare coordinates $t_{p}, r_{p}$ smoothly cover both the region inside and outside the black hole horizon.

The black hole $(2.2)$ has Hawking temperature $T_{H}=1 /(2 \pi \ell)$, while the same spacetime viewed in Poincare coordinates has zero temperature. This is analogous to the statement that Rindler space has nonzero temperature and can be understood as follows. The dual field theory will be discussed in detail in the following sections, but for now it suffices to note that the metric at infinity in the black hole spacetime is conformal to the static cylinder (1.3). Starting with Euclidean space $d s^{2}=d x^{2}+d y^{2}+d z^{2}+z^{2} d \theta^{2}$ and rescaling by $\ell^{2} / z^{2}$ yields $d s^{2}=\ell^{2}\left[d \theta^{2}+d \sigma^{2}\right]$ which is the static cylinder at temperature $T_{H}=1 /(2 \pi \ell)$. So for any conformal field theory, the standard Minkowski vacuum is equivalent to a thermal state on this static cylindert.

As mentioned in the introduction, a curve of constant Poincaré radius $r_{p}=R$ corresponds to an infalling trajectory in the black hole: $r^{2}(t)=R^{2} e^{-2 t / \ell}+\ell^{2}$. This allows

4 We thank J. Maldacena for suggesting this argument. 
us to construct a simple model of matter collapsing to form the black hole. Consider a static spherical shell of D3-branes. The metric inside the shell is ten dimensional flat spacetime, while the metric outside is $A d S_{5} \times S^{5}$. If we again introduce Milne coordinates and compactify the hyperbola, this metric becomes

$$
d s^{2}=h^{-1}\left[-d t_{p}^{2}+t_{p}^{2} d \sigma^{2}\right]+h\left[d r_{p}^{2}+r_{p}^{2} d \Omega_{5}\right]
$$

where

$$
h\left(r_{p}\right)=\frac{\ell^{2}}{r_{p}^{2}}\left(r_{p}>R\right), \quad h\left(r_{p}\right)=\frac{\ell^{2}}{R^{2}} \quad\left(r_{p}<R\right)
$$

where $R$ is the radius of the shell in Poincare coordinates. In terms of the black hole interpretation of the exterior spacetime, the shell starts at large $r$ and collapses to $r=0$ forming a horizon at $r=\ell$.

Note that the singularity in (1.1) at $t_{p}=0$ that appears after compactifying the hyperbola is naturally viewed as a cosmological singularity in AdS since it is spacelike and goes out to infinity in finite time. Since this metric is equivalent to $(2.2)$, we are led to the surprising conclusion that there is no invariant distinction between black hole singularities and cosmological singularities in this case. Although this singularity is not a curvature singularity, the slightest perturbation will cause the curvature to diverge.

There is a one parameter family of hyperbolic black holes which generalize (2.2). The metric again takes the form (2.2) where now

$$
f(r)=\frac{r^{2}}{\ell^{2}}-1-\frac{\mu}{r^{2}}
$$

These are all solutions to Einstein's equation with negative cosmological constant.5 These spacetimes describe static black holes with an event horizon at the largest zero of $f$ and a singularity at $r=0$. The constant $\mu$ is a free parameter which is related to the mass of the black hole by 6

$$
M=\frac{3 \mu \hat{V}}{16 \pi G_{5}}
$$

5 For a more detailed discussion of these solutions, see [4].

6 If one computes this mass using the boundary stress tensor this mass is shifted by a ( $\mu$ independent) constant [4]. Since we are interested in dynamical questions, the zero point of the energy is not important and we will ignore it. 
where $\hat{V}$ is the dimensionless volume of $\Sigma$. These solutions have the unusual property that there is a horizon even when $\mu$ is negative provided $\mu \geq \mu_{\text {ext }}$ with

$$
\mu_{e x t} \equiv-\frac{\ell^{2}}{4} \Rightarrow M_{e x t}=-\frac{3 \hat{V} \ell^{2}}{64 \pi G_{5}}
$$

For $\mu>0$, the singularity at $r=0$ is spacelike (like Schwarzschild-AdS), but for $\mu_{\text {ext }} \leq$ $\mu<0$, it is timelike (like Reissner-Nordstrom-AdS). In terms of the horizon radius, $r_{+}$, the Hawking temperature of these black holes is

$$
T_{H}=\frac{2 r_{+}^{2}-\ell^{2}}{2 \pi \ell^{2} r_{+}}
$$

In the extremal limit when $\mu=\mu_{\text {ext }}$, the horizon radius is $r_{+}=\ell / \sqrt{2}$ and $T_{H}=0$. For $\mu<\mu_{\text {ext }}$ there is a naked singularity.

We believe that one can form these black holes with nonzero mass by throwing in spherical shells of D3-branes with various energies. However, it is difficult to find exact ten dimensional supergravity solutions in this case since the shell will radiate. This does not occur when $\mu=0$ since the shell is following the orbit of a Killing field (Poincare time translations). There is no danger of violating cosmic censorship by throwing in a shell and forming a naked singularity, since we will see in the next section that if the energy is sufficiently negative, the shell will bounce, and never reach $r=0$.

We have seen that the $\mu=0$ black hole can be described in Schwarzschild coordinates or Poincare-like coordinates. For our later analysis, it will be very useful to work with a hybrid system of coordinates which keep the nice time slices of the Poincare coordinates, but for which the boundary theory naturally lives on the static cylinder. These are given by

$$
\tilde{r}=-\frac{r_{p} t_{p}}{\ell} \quad \tilde{t}=-\ell \ln \left(-\frac{t_{p}}{\ell}\right)
$$

(Note that $\tilde{r}$ is the same as the Schwarzschild radial coordinate $r$.) In these coordinates, the metric for the $\mu=0$ black hole is

$$
d s^{2}=-\left(\frac{\tilde{r}^{2}}{\ell^{2}}-1\right) d \tilde{t}^{2}+\tilde{r}^{2} d \sigma^{2}+\frac{\ell^{2}}{\tilde{r}^{2}} d \tilde{r}^{2}+\frac{2 \ell}{\tilde{r}} d \tilde{t} d \tilde{r}
$$

These coordinates extend smoothly across the horizon. 


\section{The Dual CFT}

\subsection{CFTs on cosmological vs. static backgrounds}

In order to study the systems discussed in the previous section non-perturbatively, we will analyze the dual CFT. This CFT lives on a non-fluctuating background spacetime which is homogeneous along hyperbolic spatial slices. Since the $\mathcal{N}=4 \mathrm{SYM}$ theory is conformally invariant, we should obtain the same results from the field theory in any conformal frame, modulo subtleties arising in the case of singular conformal transformations. There are two conformal frames which will prove useful to consider, as discussed in [14]. First, consider the CFT on the spacetime (1.2)

$$
d s^{2}=-d t_{p}^{2}+t_{p}^{2} d \sigma^{2}
$$

with $d \sigma^{2}$ the metric on $\mathbb{H}_{3}$. This is simply a hyperbolic slicing of flat spacetime, and has vanishing curvature.

In the presence of additional stress-energy which is homogeneously distributed along the hyperbolic slices, however, the system becomes singular as $t_{p} \rightarrow 0$. Upon orbifolding by $\Gamma$, so that $d \sigma^{2}$ is the metric on $\Sigma$, then even in the absence of additional sources of stress energy, the spacetime (3.1) has a Milne-like singularity at $t_{p}=0$. The dual bulk geometry is the region of spacetime covered by the metric (1.1).

Next, consider the cooordinate transformation of (3.1) (with $t_{p}<0$ ) to conformal time $\eta$ :

$$
t_{p}=-\ell e^{-\eta / \ell}
$$

which yields $d s^{2}=e^{-2 \eta / \ell}\left(-d \eta^{2}+\ell^{2} d \sigma^{2}\right)$. We now conformally rescale the metric:

$$
d s_{\text {static }}^{2}=e^{2 \eta / \ell} d s^{2}=-d \eta^{2}+\ell^{2} d \sigma^{2}
$$

and the operators of the CFT. This maps the CFT on (3.1) to that on (3.3). This trans-

formation is singular at $t_{p}=0$. The static metric $d s_{\text {static }}^{2}$ has constant negative scalar curvature $\mathcal{R}$ coming from the hyperbolic spatial slices. This static background spacetime has no singularity whether or not we compactify the space or include homogeneous sources. We will argue below that the singularity in the spacetime physics will manifest itself in this static background through the behavior of the rolling scalar fields of the CFT. 


\subsection{Yang-Mills theory and scalar dynamics}

The $\mathcal{N}=4$ SYM theory has six adjoint scalar fields $\Phi^{i}$, which together with the gauge fields are governed by the classical action

$$
\mathcal{S}_{S Y M}=\frac{-1}{g_{Y M}^{2}} \int \sqrt{-g} \operatorname{Tr}\left(F^{2}+(D \Phi)^{2}+\left[\Phi^{i}, \Phi^{j}\right]^{2}+\frac{1}{6} \mathcal{R} \Phi^{2}\right)+\text { fermions }
$$

These scalars, and their gravity-side manifestation as collective coordinates for D3-branes, will play a key role in our analysis. The commutator interaction among the scalar fields is minimized by commuting matrices, i.e. eigenvalues $\phi_{a}^{i}, a=1, \ldots N$ of the $\Phi^{i}$. In the case of flat spacetime, this leads to a moduli space of scalar field VEVs, homogeneous along flat spatial slices; otherwise generically the moduli space is only approximate. The off-diagonal modes of the $U(N)$ adjoint matrices (the "W bosons") have masses

$$
m_{W, i a b}^{2} \sim g_{Y M} \sum_{j \neq i}\left(\phi_{a}^{j}-\phi_{b}^{j}\right)^{2}
$$

They all become light at the origin $\phi_{a}^{i} \rightarrow 0$.

Consider first this quantum field theory on (3.1). This is a CFT on a FriedmannRobertson-Walker background or "collapsing cone" with scale factor $a\left(t_{p}\right)=t_{p}$. For large $\left|t_{p}\right|, H=\frac{\dot{a}}{a} \sim 1 / t_{p}$ is small and the physics is smooth. For small $\left|t_{p}\right|$, there is a singularity; Kaluza-Klein modes on the hyperboloid blueshift without bound and the ratio $m_{W} / m_{K K} \propto\left|t_{p}\right|$ goes to zero.

Next, let us analyze this system in the static frame (3.3). The conformal transformation acts on the scalar fields as

$$
\Phi=e^{-\eta / \ell} \Phi_{p}
$$

where $\Phi$ refers here to the field in the static conformal frame. Applying this to a diagonal element of $\Phi$, we see from (3.5) that in this frame $m_{W} \rightarrow 0$, as the scalar field reaches the origin, while the KK mass is constant. Thus, both frames contain a regime of the dynamics in which the dimensionless ratio $m_{W} / m_{K K} \propto\left|t_{p}\right|$ goes to zero in this limit, and these regimes should be identified.

In the static frame, the conformal coupling of the scalar field to the scalar curvature $\mathcal{R}$ gives a negative mass squared to the scalars. The theory does not have a ground state. Nonetheless, the gauge theory is well defined. First, only a finite number of modes (and in

7 Of course, the theory on the collapsing cone does not have a unique vacuum either. 
some cases, only the zero modes [15]) of the scalars experience this instability. Secondly, it takes an infinite time for these zero modes to roll down a $-\operatorname{Tr} \Phi^{2}$ potential to infinity. The classical and quantum evolution is well defined for any initial state. Finally, the physics of the scalars rolling off to infinity is equivalent to the scalars being constant at late time on the expanding cone, a regime which is completely well behaved.

We deduce that in the static frame (3.3), the physics of the singularity of the metric (3.1) corresponds to scalar fields rolling toward the origin $\Phi=0$ and scattering off a quadratic potential barrier. Since both the collapsing cone metric and the conformal transformation to the static frame that we used are singular, we will focus on the field theory formulated in the static frame, and study the relationship between the spacetime and the field theory pictures.

To begin with, let us consider gauge theory states at different energies $E$, and map them to the corresponding family of backgrounds on the gravity side. For energies $E>0$, one can argue that the generic state of the field theory will be an excited gas of W-bosons and Kaluza-Klein modes, with the scalars trapped for a long time at the origin. The gravitational duals will be the $M>0$ black holes. Negative energies corresponding to $M<0$ can be accessed by lowering the energy of the zero modes of the scalar fields; the potential barrier due to the curvature energy means that the theory will be out on the Coulomb branch. More generally, as noted in $\S 2$, we can create black holes at all energies, by studying shells of N D3-branes wrapped along $\Sigma$, and distributed evenly over the $S^{5}$. Out on the Coulomb branch, the eigenvalues of $\Phi$ are naturally associated with the radial positions of the dual D-branes.

We find a simple correspondence between the quantum corrections in the scalar field theory and the causal structure in the gravitational duals. In the static frame, the eigenvalues for the scalar fields see an inverted harmonic oscillator potential. If the off-diagonal modes are set to zero (so that the commutator term in (3.4) does not contribute), then there are classical trajectories in which each eigenvalue $\phi$ of energy $E_{\phi} \sim-\phi_{0}^{2} \ell / g_{Y M}^{2}$ executes a motion

$$
\phi(t)=\phi_{0} \cosh \left[\left(\eta-\eta_{0}\right) / \ell\right]
$$

with $\phi$ bouncing off the inverted harmonic oscillator potential at a minimal value $\phi=\phi_{0}$ when $\eta=\eta_{0}$. With $N$ eigenvalues moving in an $S O(6)$ invariant configuration, this has energy

$$
E \sim-\frac{N \phi_{0}^{2} \ell}{g_{Y M}^{2}}
$$


Near the origin, the off-diagonal modes of the $U(N)$ matrices become light, and generate quantum mechanical effects which drastically affect the evolution. At weak coupling $(\lambda \ll 1)$, particle production of off-diagonal modes ("W bosons") traps the field at $\phi=0$ [9,11]. The strength of this effect is controlled by

$$
\frac{1}{\phi^{2}}\left|\frac{d \phi}{d \eta}\right| \sim \frac{1}{\phi_{0} \ell}
$$

At strong 'tHooft coupling, the leading effect is the renormalization of the action due to loops of $\mathrm{W}$ bosons, which sum up to the DBI action and slow and trap the field at $\phi=0$ [10]. The figure of merit for the importance of loop effects arising from the time dependence in the rolling scalar background 8 is $\sqrt{\lambda}$ times $(3.9)$ :

$$
\frac{\sqrt{\lambda}}{\phi^{2}}\left|\frac{d \phi}{d \eta}\right| \sim \frac{\sqrt{\lambda}}{\phi_{0} \ell}
$$

For sufficiently negative energy $E$ the field bounces off the inverted harmonic oscillator potential at a field value $\phi_{0}$ sufficiently large that these effects never become important. The loop effects become important when the minimal field value is small enough that (3.10) becomes $\geq 1$, i.e. when $\phi_{0} \ell \leq \sqrt{\lambda}$. Translating this into an energy scale using (3.8), this implies $E>-N^{2} / \ell$. This energy scale is significant on the gravity side: from (2.9) and the fact that $\frac{\ell^{2}}{G_{5}} \sim \frac{N^{2}}{\ell}$ we see that it is parametrically the energy scale above which there is a black hole horizon in the dual geometry. Therefore the field theory produces nontrivial DBI corrections precisely in the regime where the classical gravity side exhibits nontrivial causal structure.9

8 In the case of present interest, the field theory is not formulated on Minkowski space and the DBI action in general also contains corrections arising from the curvature of the background metric and from finite temperature effects.

9 In [16], the authors have argued that for Schwarzschild observers, the breakdown of the DBI action of D-brane probes at the horizon coincides with the appearance of light states connecting the probe and the hot Dp-branes. It would be interesting to see if a similar picture here led to thermalization of a collapsing shell as it approached the horizon. 


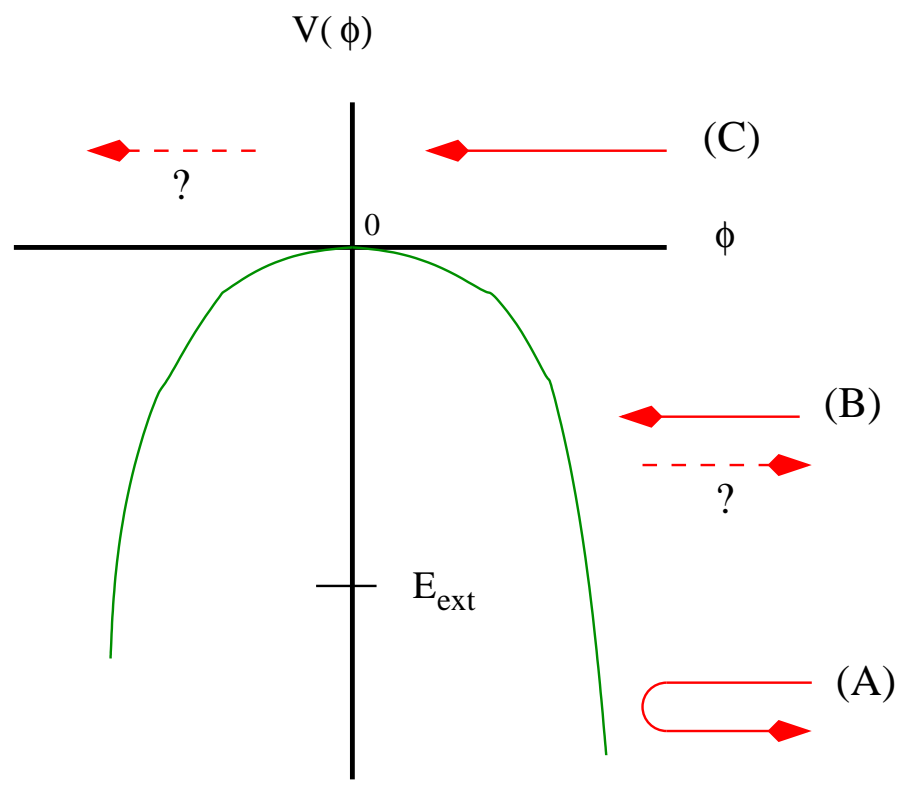

Fig. 2: Phases of the scalar field dynamics. The curve is the inverted harmonic oscillator potential, the straight lines are the incoming scalar field trajectories at various energies, and the dashed lines are the classical late time behavior (meant to be at the same energy) without quantum corrections. Trajectory (A) corresponds to a bouncing scalar. In trajectory (B), the scalar would bounce without quantum corrections, but DBI corrections slow the scalar near the potential barrier and quantum corrections become important. In trajectory $(\mathrm{C})$, the scalar would sail over the potential barrier without quantum corrections, but again DBI and other quantum corrections become important near the origin.

In general, the dynamics of the scalar fields have a phase structure which matches the different causal structures for the dual spacetimes, as seen in Figures 2 and 3. Let us collect the above statements to make this clear. For $E<-N^{2} / \ell$, the scalar fields bounce off of the potential before quantum effects are important. The dual spacetime has no horizons, and we believe that the naked singularity is removed by the D-brane shell along the lines of [17]. For $-N^{2} / \ell<E<0$, the scalar field trajectories see strong quantum corrections before they reach the potential barrier, roughly at the location of the horizon; furthermore, the barrier prevents them from reaching the origin. The dual spacetimes are black holes of Reissner-Nordstrom type, with inner and outer horizons cloaking timelike singularities (although the inner horizon is unstable to forming a singularity). Finally, for $E>0$, the scalar field trajectory continues to be corrected when the scalars reach the horizon; furthermore, there is no barrier preventing them from reaching the origin. In this regime, the dual spacetimes are black holes with spacelike singularities. 


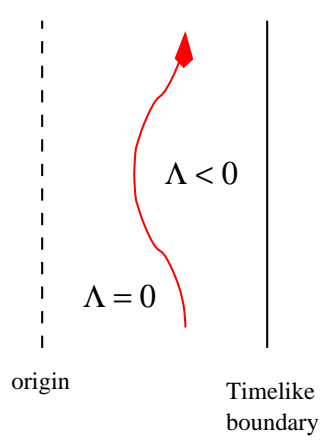

(A)

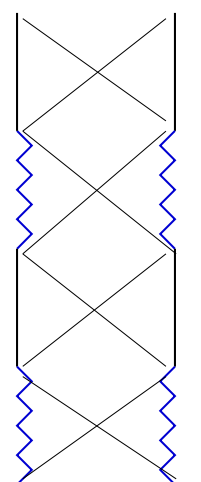

(B)

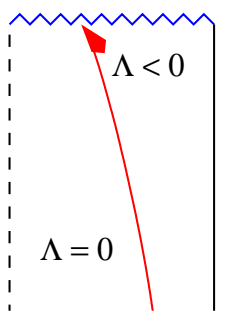

(C)

Fig. 3: Causal structures of classical black holes formed by scalar trajectories shown in the previous figure. For case (A), the brane bounces and the putative naked singularity in (2.2) for $E<E_{\text {ext }}$ is screened. For (B) we are unsure of the trajectory of the collapsing shell, and have shown the Reissner-Nordstrom-like causal structure of the black holes (2.2) for $0>E>E_{\text {ext }}$. For (C) the black holes have spacelike singularities, and we have shown the diagram for a $M=0$ black hole.

The stalling of the system at small values of $\phi$ appears to be key to understanding the resolution of the singularity and the description of the decay of the black holes (2.2). There are many coupled features of the physics in this regime, which we will only partially control. These include the loop effects discussed above, production of KK modes and W bosons, the Casimir energy (which in general breaks hyperbolic symmetry [18), the dynamics of Wilson lines (both continuous and discrete), and the spreading of the wave function for the scalars.

In the present work, we will largely focus on the CFT description of the process of an observer propagating through the horizon. The first step at large $\lambda$ is to take into account the effects of the DBI action. This will lead us to a scheme for describing physics inside the horizon, accessible before reaching the full complications of the singularity. In particular, in order to analyze the dynamics of the scalar fields at strong coupling, we can make use of the gravity side of the AdS/CFT correspondence in the regime where it is effective (i.e. away from the singularity). Let us turn now to these issues. In $\S 5$, we will explore the physics of the singularity and the fate of the black holes in light of its relation to the dynamics of the Yang-Mills theory at the origin of moduli space.

\section{How to look behind the horizon}

For gauge theories with a gravitational dual, the theory on a static $4 \mathrm{~d}$ spacetime at finite temperature is taken to describe the experience of a "Schwarzschild" observer in the 
dual 5d static black hole spacetime. Classically, such an observer cannot see behind the horizon. When the black hole is formed dynamically, unitarity requires that the experience of infalling observers behind the horizon is measured by a Schwarzschild observer through subtle correlations in the black hole's Hawking radiation. 10

The sections above give a prescription for describing observers such as D3-brane probes falling through the horizon of $M=0$ hyperbolic black holes: study the gauge theory dual to the $t_{p}<0$ half of the Poincaré patch. How can we relate this to the experience of a Schwarzschild observer? In this section, we give an answer for infalling D3-brane probes wrapping the hyperbolic space $\Sigma$. The essential point is that one may promote the time direction to a field variable of the D-brane action, which amounts to adding an additional gauge invariance (invariance under worldvolume reparametrizations). The resulting DBI effective action for the D3-brane probe is invariant under target space reparametrizations. One can transform the theory from a description suited to different observers by simple field redefinition. This answer generalizes readily to $M \neq 0$ black holes, and to black holes with other horizon topologies, such as those with spherical or flat horizons which appear in asymptotically AdS backgrounds.

We will start in $\S 4.1$ by describing the the DBI actions for D3-brane probes according to both "infalling" and "Schwarzschild" observers, and constructing a probe action for infalling observers which is dual to a non-singular gauge theory. In $\S 4.2$ we will discuss the change of variables of the probe theory which transforms these actions into each other. These maps take the form of a field-dependent reparametrization of the gauge theory time variable. In $\S 4.3$ we will argue that these reparametrizations may arise from a change of gauge in the underlying Yang-Mills theory.

\subsection{The effective action for D3-brane probes}

Consider a single D3-brane wrapping $\Sigma=\mathbb{H}_{3} / \Gamma$, with the worldvolume time set equal to the Poincaré time and the position described by the radial direction in Poincaré coordinates. The DBI action for this D3-brane probe is:

$$
S_{d b i p}=\frac{\hat{V}}{g_{s}\left(\alpha^{\prime}\right)^{2}} \int d t_{p}\left[\frac{r_{p}^{3} t_{p}^{3}}{\ell^{3}} \sqrt{\frac{r_{p}^{2}}{\ell^{2}}-\frac{\ell^{2}}{r_{p}^{2}} \dot{r}_{p}^{2}}-\frac{r_{p}^{4} t_{p}^{3}}{\ell^{4}}\right]
$$

10 Different language describes the consequences of unitarity for "eternal" black holes, whose field theory dual is a tensor product of two copies of the zero-temperature field theory [19,20,21,22, coupled via a correlated "thermal" state. 
(Here $\hat{V}$ is the volume of the compact hyperbolic space). Gauge-gravity duality tells us that (4.1) arises from the Yang-Mills theory on the cosmological spacetime (3.1), with one eigenvalue $\phi_{p}=m_{s}^{2} r_{p}$ of the adjoint scalar taken far out along the Coulomb branch, breaking $U(N) \rightarrow U(N-1) \times U(1)$; it is the effective action for this eigenvalue, which arises from integrating out the massive $\mathrm{W}$ bosons with mass $m_{W}=\phi_{p}$. In particular, one might have deduced this action by considering the AdS/CFT correspondence in Poincaré coordinates, and performing the orbifold on that theory, truncating to zero modes after the coordinates have been changed and the orbifold projection has been performed.

This action is well suited to describing an infalling observer (where the observer is our D3-brane probe). The equation of motion for $r_{p}$ is:

$$
4 \frac{r_{p}^{3}}{\ell^{4}}\left(\frac{1}{\gamma_{p}}-1\right)+\frac{3 \dot{r}_{p} \gamma_{p}}{t_{p}}+\frac{d}{d t_{p}}\left(\dot{r}_{p} \gamma_{p}\right)+2 \frac{\dot{r}_{p}^{2}}{r_{p}} \gamma_{p}=0
$$

where $\gamma_{p}=1 / \sqrt{1-\ell^{4} \dot{r}_{p}^{2} / r_{p}^{4}}$. This has a constant- $r_{p}$ solution which describes a D3-brane falling into the black hole, reaching the singularity at $t_{p}=0$, which is at finite time. The second term here contains the effects of Hubble friction on the motion, following from the homogeneity along the hyperbolic as opposed to flat spatial slices of the Poincaré slices of $A d S_{5}$. While DBI corrections may begin to become important near the horizon, it is easy to check that fluctuations around this solution remain under control all the way through the horizon. As we will discuss at the end of this section, it is useful to describe this in a static conformal frame obtained by the conformal transformation (3.3) to the static cylinder; this leads to a description of infalling observers related to the coordinates $\tilde{r}, \tilde{t}$ (2.11).

On the other hand, we also wish to describe an observer who remains outside of the black hole, and so study the detailed implementation of black hole complementarity from the field theory point of view. Furthermore, as stated in the introduction of this section, past presentations of black holes are in terms of a gauge theory on a static background at finite temperature, which describes Schwarzschild observers, and we would like to study physics behind the horizons of these black holes as well.

Consider then a D3-brane wrapping $\Sigma$, with worldvolume time equated with Schwarzschild time, and the position described by the radial position in Schwarzschild 
coordinates. The DBI action describing the spacetime dynamics of this probe is 11

$$
S_{d b i s}=-\frac{\hat{V}}{g_{s}\left(\alpha^{\prime}\right)^{2}} \int d t\left[r^{3} \sqrt{f(r)-\frac{\dot{r}^{2}}{f}}-\frac{\left(r^{4}-\ell^{4}\right)}{\ell}\right]
$$

Gauge-gravity duality tells us that this action arises from considering $\mathcal{N}=4 U(N)$ YangMills theory on (3.3) at finite temperature, with one eigenvalue $\phi=m_{s}^{2} r$ of the adjoint scalar taken out onto the Coulomb branch, again breaking the $U(N)$ gauge symmetry to $U(N-1) \times U(1) . S_{d b i s}$ is the effective action for this eigenvalue, arising from integrating out the W-bosons (transforming in the fundamental of the unbroken $U(N-1)$ ) with mass $m_{W}=\phi$.

Near the horizon, the scalar field approaches its speed limit

$$
\dot{r}^{2} \rightarrow f(r)^{2}
$$

and the relativistic $\gamma$ factor $\gamma=1 / \sqrt{1-\dot{r}^{2} / f^{2}}$ approaches infinity. Therefore, the probe takes an infinite Schwarzschild time to reach the horizon, as is clear from the time slicings depicted in Figure 1b. Furthermore, perturbations about the background solution become large near the horizon, for the following reason. Expand $r \equiv r_{0}(t)+\delta r$ about a spatially homogeneous classical solution $r_{0}(t)$. The resulting effective action for the perturbations $\delta r$, obtained by expanding the square-root action, has a quadratic term scaling like $\gamma^{3}$, cubic interactions scaling like $\gamma^{5}$, and so on. Therefore the interactions among the perturbations $\delta r$ become strong as the brane approaches the horizon. This suggests that (4.3) is not a good effective description near the horizon, and one should choose a different set of variables to describe the probe dynamics.

Such variables are provided by our original Poincaré description. The reader may worry that as we originally formulated it, the gauge theory dual which describes infalling observers well lives on a singular spacetime. In fact, we can choose field theory variables for the gauge theory on the static cylinder which sees behind the horizon, giving us the best of both worlds. Furthermore, this set of fields will be most readily generalized to other black holes.

11 The last term comes from $\int A_{4}$ where $A_{4}$ is the potential for the five-form flux. In Schwarzschild coordinates, the potential which is nonsingular on the horizon is $A_{4} \propto\left(r^{4}-\ell^{4}\right) d t \wedge \epsilon_{3}$ (where $\epsilon_{3}$ is the volume form on the hyperboloid). Starting with the form of $A_{4}$ in Poincare coordinates and applying the coordinate transformation (2.1) yields an expression for $A_{4}$ which agrees with this up to a term $d A_{3}$ where $A_{3}$ is finite on the horizon. 
These variables are just the tilded coordinates introduced at the end of $\S 2$. The DBI action in the metric (2.12) is

$$
S_{d b i p}^{\prime}=-\frac{\hat{V}}{g_{s}\left(\alpha^{\prime}\right)^{2}} \int d \tilde{t} \frac{\tilde{r}^{4}}{\ell}\left\{\sqrt{1-\frac{\ell^{4}}{\tilde{r}^{4}}\left(\frac{d \tilde{r}}{d \tilde{t}}+\frac{\tilde{r}}{\ell}\right)^{2}}-1\right\}
$$

Since (3.2),(3.3) map the collapsing cone to the static cylinder, this theory should also arise from Yang-Mills on the non-singular static cylinder.

In these coordinates, the late-time solutions approach the speed limit

$$
\left(\frac{d \tilde{r}}{d \tilde{t}}+\frac{\tilde{r}}{\ell}\right)^{2} \rightarrow \frac{\tilde{r}^{4}}{\ell^{4}}
$$

In other words, all solutions asymptote to $\tilde{r} \rightarrow \tilde{r}_{0} e^{-\tilde{t} / \ell}$ at late times $\tilde{t}$. In these variables, the classical approximation breaks down at the singularity: as in (4.1), the evolution through the horizon is smooth and classical. This should not surprise us: the transformation (2.11) reparametrizes the equal-time slices in Poincaré coordinates slice by slice, but does not change the slicing. The upshot is that we can separate the problem of the spacetime singularity from the problem of studying Yang-Mills theory on a nonstatic, singular spacetime.

The reader may also object that we have argued in $\S 3$ that the physics of the singularity is captured by the origin of moduli space, which the Schwarzschild coordinate $r(t)$ cannot classically reach. Black hole complementarity states that the Schwarzschild observer should somehow be able to access the physics near the singularity, if only through subtle correlations in the radiation emitted by the black hole. Moreover, it would be worrisome if a regime of field space was somehow excised. However, we believe that while the classical D3-brane probe reaches the horizon only as $t \rightarrow \infty$, these probes and the field theory degrees of freedom which couple to them become strongly fluctuating in Schwarzchild variables. Such fluctuations may extend to the origin of moduli space; but they will have no classical spacetime interpretation. A Schwarzschild observer will see infalling objects approach the horizon and thermalize.12

In $\S 4.2$, we will describe the transformation of the quantum variables of the low energy effective action, which maps (4.1) to (4.3). However, the full Yang-Mills theory on the static cylinder seems to produce two effective actions. On the one hand, the Schwarzschild DBI

12 It is possible that a story analogous to [16] pertains here. 
action (4.3) seems natural for a theory on the static cylinder at finite temperature, by analogy with other black holes. On the other hand, since (3.2)(3.3) map the Yang-Mills actions on (3.1),(3.3) into each other, it would appear that (4.5) should arise naturally from the Yang-Mills theory. This is not a contradiction: the definitions of time in (4.3),(4.5) differ for finite field values. Furthermore, the form of the effective action will depend on the coordinates used on the space of eigenvalues, and on the choice of gauge used (since the eigenvalues are not gauge-invariant objects). In $\S 4.3$ we will argue that that the difference between (4.3) and (4.5) arises at least in part from the action of (3.2)(3.3) on the gauge condition used to compute the DBI action.

\subsection{Time reparameterizations in quantum mechanics}

The basic idea behind the transformation of quantum variables describing the Schwarzschild and infalling observers is simple. Beginning with either (4.1), (4.3), or (4.5), one may promote the corresponding times $t_{p}, t$ to quantum variables $t_{p}(\tau), t(\tau)$ at the price of introducing a gauge invariance - worldvolume reparametrizations - which must be dealt with in the path integral. The resulting DBI action of a particle is written as:

$$
S \propto \int d \tau \sqrt{-\operatorname{det} G_{\mu \nu} \partial_{\tau} X^{\mu} \partial_{\tau} X^{\nu}}+S_{W Z}
$$

where $S_{W Z}$ is the "Wess-Zumino" coupling to background Ramond-Ramond fields. In addition to the aforementioned gauge invariance, this action is also manifestly invariant under target space coordinate transformations. 13 In particular, (4.3) becomes:

$$
S_{d b i s c}=-\frac{\hat{V}}{g_{s} \ell_{s}^{4}} \int d \tau\left[r^{3} \sqrt{f t_{, \tau}^{2}-\frac{r_{, \tau}^{2}}{f}}-\frac{\left(r^{4}-\ell^{4}\right) t_{, \tau}}{\ell}\right]
$$

and (4.5) becomes:

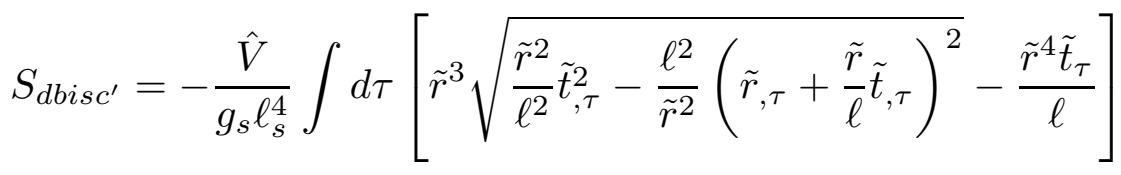

The transformation between (4.8) and (4.9) is clear. Since (4.7) is invariant under target space coordinate transformations, we need merely find the coordinate transformation

13 Up to boundary terms, which can be removed by gauge transformations of the RamondRamond fields. 
that relates the spacetime coordinates $r, t$ to $\tilde{r}, \tilde{t}$, and promote these to field redefinitions. These transformations are:

$$
\tilde{r}=r ; \quad \tilde{t}=t+\frac{\ell}{2} \ln \left(1-\frac{\ell^{2}}{r^{2}}\right) .
$$

In the covariant DBI action (4.7), this is just a (complicated) field redefinition. After transforming the action, one may fix gauge again by selecting a time variable and setting it equal to $\tau$. In particular, (4.3) arises by writing (4.7) in the form (4.8) and setting $t=\tau$; (4.5) arises by writing (4.7) in the form (4.9) and setting $\tilde{t}=\tau .14$

From the point of view of the gauged-fixed actions (4.3),(4.5), these transformations look exotic. $r, \tilde{r}$ are quantum observables, so that the second equation in (4.10) appears to be an operator-valued reparametrization of the time variable. On the other hand, in theories with reparametrization invariance, this kind of transformation is common. For example, when computing the density fluctuations which arise during inflation, one can choose spatially flat equal-time slices and study quantum fluctuations of the inflaton; or one may choose a gauge in which the inflaton fluctuations vanish and a scalar mode of the spatial metric fluctuates. These are related by a field-dependent change of the equal-time slicing.

Such field redefinitions and gauge transformations leave the physics invariant. In practice, they will turn a simple correlation function in one set of variables into a correlation function of very complicated operators in the other set of variables. The field redefinitions and gauge transformations we perform will change which set of operators are natural to study. Certain questions will be naturally answered in a frame for which the corresponding fields are well described by a classical limit. For observers falling into the black hole, the appropriate frame is realized by the Poincaré coordinates and associated DBI actions (4.1)(4.5), which describe semiclassical evolution of a probe brane through the horizon.

There has been speculation that to follow an object falling through a black hole horizon, one should evolve the probe with a new Hamiltonian in the gauge theory. This is indeed what happens in the familiar case of crossing the Poincaré horizon by transforming from Poincare to global coordinates. The Hamiltonian in the gauge theory on $S^{3} \times \mathbb{R}$ is

14 Of course, (4.3) and (4.5) arise from a wrapped D3-brane, so that one must start with a 3+1-dimensional DBI action and fix the gauge for spatial reparametrizations as well; however, since the spatial directions along $\Sigma$ do not figure into our coordinate transformations, we will ignore this step. 
related to the dilatation operator in Minkowski spacetime. However there is a key difference between this and the case we are considering here: The choice of time on the boundary changes when we transform Poincaré into global coordinates. In our present example, the choice of time on the static cylinder does not change. The constant $\eta$ surfaces on the boundary can be extended inside the bulk spacetime either along constant $t$ or constant $\tilde{t}$ time slices.

In the present case, despite the nontrivial change in bulk time slices, the Hamiltonians in the frames $(4.3),(4.5)$ are the same. To see this, we can use (4.8) and (4.9) to compute the conjugate momenta. It is easy to show that:

$$
\begin{aligned}
& p_{\tilde{r}}=p_{r}-\frac{\ell}{r f(r)} p_{t}+\frac{r^{4}-\ell^{4}}{\ell^{4} r f(r)} \hat{V} N \\
& p_{\tilde{t}}=p_{t}+\frac{\hat{V} N}{\ell} .
\end{aligned}
$$

While the momenta conjugate to $r, \tilde{r}$ transform nontrivially into each other, $p_{t}$ and $p_{\tilde{t}}$ are equal up to an overall constant shift. Now upon choosing the gauge $t=\tau,-p_{t}$ becomes the Hamiltonian derived from (4.3); while choosing the gauge $\tilde{t}=\tau,-p_{\tilde{t}}$ becomes the Hamiltonian derived from (4.5). Eq. (4.11) shows that these Hamiltonians are the same up to a constant which is unimportant for the field theory dynamics.

On the other hand, because $p_{r}$ and $p_{\tilde{r}}$ do transform nontrivially, the functional form of the Hamiltonians will be quite different in different frames. The reparametrization invariance implies a nontrivial constraint on the canonical variables. This can be written as a relation between the conjugate momentum $p_{t}$ and the variables $p_{r}, r$. Using the fact that $p_{t}$ is minus the Hamiltonian (in the gauge $\tau=t$ ) we find:

$$
H\left(p_{r}, r\right)=\frac{\hat{V}}{g_{s}\left(\alpha^{\prime}\right)^{2}}\left[f(r) \sqrt{\left(\frac{g_{s}\left(\alpha^{\prime}\right)^{2} p_{r}}{\hat{V}}\right)^{2}+\frac{r^{6}}{f}}-\frac{\left(r^{4}-\ell^{4}\right)}{\ell}\right]
$$

This can also be obtained by computing the Legendre transformation of (4.3) to find the Hamiltonian directly. Similarly, using the fact that $p_{\tilde{t}}$ is minus the Hamiltonian in the gauge $\tilde{t}=\tau$, we find:

$$
H\left(p_{\tilde{r}}, \tilde{r}\right)=\frac{\hat{V}}{g_{s}\left(\alpha^{\prime}\right)^{2}}\left[\frac{\tilde{r}^{2}}{\ell^{2}} \sqrt{\left(\frac{g_{s}\left(\alpha^{\prime}\right)^{2} p_{\tilde{r}}}{\hat{V}}\right)^{2}+\ell^{2} \tilde{r}^{4}}-\frac{\tilde{r}}{\ell} p_{\tilde{r}}-\frac{\tilde{r}^{4}}{\ell}\right]
$$


Note that the form of $H$ in terms of the new canonical variables $p_{\tilde{r}}, \tilde{r}$ is different. It would be interesting to pursue these transformations further, for example by comparing the evolution of wavepackets in each set of coordinates.

An important question is how these transformations lift to the full set of variables of the underlying Yang-Mills theory. Since the non-abelian generalization of the DBI action is not known beyond low orders in velocities, this may prove somewhat difficult. We now turn to an alternate route to understanding the transformation of the full Yang-Mills theory.

\subsection{Coordinate transformations vs. gauge transformations}

The Yang-Mills actions on (3.3) and (3.1) are related by a conformal transformation. The DBI action (4.1) transforms under this conformal transformation to (4.9) (with $\tau=\tilde{t}$ ), not to (4.3): one requires the additional field-dependent coordinate transformation (4.10) to pass from (4.5) to (4.3).

On the other hand, the effective action (4.3) naturally captures thermalization in the gauge theory, and it is clear that any calculation using a gauge, regulator, and background field configuration which respects the time-reversal symmetry of the Yang-Mills theory and of the thermal state cannot produce (4.5) as an effective action. For example, the expansion in $(\ell / \tilde{r})^{2} \sim \lambda /(\ell \tilde{\phi})^{2}$ becomes a power series in $\left(\dot{\tilde{r}}+\frac{\tilde{r}}{\ell}\right)^{2}$ rather than $\dot{\tilde{r}}^{2}$.

So either one of $(4.3),(4.1)$ does not arise from the Yang-Mills theory on (3.3),(3.1) respectively, or additional input is required to derive a particular form of the DBI action as an effective action. An important input is the choice of $U(N)$ gauge. The form of the 1PI effective action depends on the choice of gauge used to compute it - only the physical quantities, computed from the 1PI action (for scattering amplitudes, this is the sum over tree graphs contributing to a process), need be invariant.

In fact, the gauge-fixing term in background field gauge is not invariant under conformally rescaling the metric. Consider the Yang-Mills theory on the cosmological spacetime (3.1). We choose as background fields a vanishing gauge field, $A_{\mu}=0$, and a scalar field profile $\bar{\Phi}^{i}$ (with $i$ an index transforming as a $\mathbf{6}$ under the $S O(6)$ R-symmetry) corresponding to a brane at a fixed location on $S^{5}$ and moving along the radial direction $r_{p}$ in the $M=0$ hyperbolic black hole spacetime. Consider the background field gauge condition 15

$$
G_{p}=\nabla^{\mu,(p)} A_{\mu}^{(p)}+i \sum_{i}\left[\bar{\Phi}_{p}^{i}, \delta \Phi_{p}^{i}\right]=0
$$

15 See also 23]. To justify the last term, note that if we were to use T-duality on a D3-brane at a point on $T^{6}$, this would become the standard $D_{\bar{A}}^{\mu} A_{\mu}=0$ background field gauge condition, where $\bar{A}$ is the background gauge field. 
where $\delta \Phi^{i}$ is the fluctuating part of $\Phi^{i}$. Due to the covariant derivative, this gauge condition is not invariant under Weyl transformations, so that:

$$
G_{p} \rightarrow e^{2 t / \ell}\left(\nabla^{\mu} A_{\mu}+\frac{2}{\ell} A_{t}+i \sum_{i}\left[\bar{\Phi}^{i}, \delta \Phi^{i}\right]\right)=e^{2 t / \ell} \tilde{G}
$$

Note that $\tilde{G}$ is not invariant under time reversal with respect to $\tilde{t}$; this makes this gauge condition a plausible choice for deriving (4.5) as an effective action.

The possibility that (4.3),(4.5) arise from the same Yang-Mills actions computed in different gauges is suggested by the related story for the action of special conformal transformations on Yang-Mills theory in $\mathbb{R}^{4}$. The Yang-Mills theory is invariant under such transformations. The DBI action for D3-brane probe in AdS is invariant under modified special conformal transformations, in which one adds a field-dependent term to the transformation of the coordinates [24]. This arises from pulling the spacetime isometry back to the worldvolume fields of the D-brane. Such a modified transformation is not an invariance of the underlying Yang-Mills action. However, the AdS/CFT correspondence states that the DBI action should arise from the Yang-Mills theory out on the Coulomb branch, after integrating out the W-bosons which become massive out on the Coulomb branch. (This has been verified to leading order in the inverse distance along the Coulomb branch |25,26.)

In [23], the authors pointed out that while the Yang-Mills action is indeed invariant under the special conformal transformation without the field-dependent term, the gauge fixing term for background field gauge is not invariant under special conformal transformations. In order to restore the gauge condition to its original form after a special conformal transformation, a field-dependent gauge transformation is required. The authors checked that to one loop, the combined action of the usual special conformal transformation and the field-dependent gauge transformation on the low-energy effective action is equivalent to the modified transformation of [24], and is indeed a symmetry of the effective action.

In $\S 4.2$ we introduced a gauge invariance - worldvolume reparametrization invariance and showed that the experience of different observers was related by a combination of field redefinition and change of how this gauge freedom was fixed. Here we have argued that these descriptions can also be related by changing the $U(N)$ gauge fixing condition. If our suggestion holds, there is a nontrivial relationship between the $U(N)$ gauge invariance and probe worldvolume plus spacetime diffeomorphisms. It would be very interesting to study this further. 


\section{Comments about the singularity}

We have seen that the hyperbolic black hole (2.2) has an equivalent description in terms of the Poincare patch. For describing evolution across the horizon, the latter description is clearly preferable since the natural time slices cross the horizon smoothly. Furthermore, a conformal transformation of this theory to the static cylinder removes the singularity in the boundary spacetime and replaces it with the physics of the scalars near the origin. In particular, the action (4.5) allows us to follow D-brane probes all the way to the singularity, at $\phi=0$. Although we cannot control all aspects of the physics in this regime, we can make the following qualitative comments.

On the static cylinder, the scalars experience a $-\operatorname{Tr} \Phi^{2}$ potential due to the negative curvature. Naively, it would appear that one could send in an eigenvalue with large positive energy and it would sail over the top of the potential. From the bulk standpoint, this would correspond to a D-brane entering the black hole, crashing through the singularity and reemerging to the future. This striking conclusion is not correct. As we have discussed, there are various effects which can slow the evolution of the scalar near the origin.

Consider first the formation of the black hole by a collapsing shell of D3-branes. Initially, the SYM scalars are diagonal, with the eigenvalues coming in from infinity. The off diagonal modes (W-bosons) are very massive and start in their ground state. As the eigenvalues approach zero, the off diagonal modes become light and are copiously produced, leading to a complicated, strongly coupled, excited state. From the bulk standpoint, as the shell of D3-branes becomes smaller, open strings are excited. A brane cannot return to large radius unless the open strings attached to it decay. This traps the eigenvalues near zero. If $\mathrm{N}$ is strictly infinite, the eigenvalues will be trapped forever. This provides a microscopic description of the formation of a classical black hole. We expect that a similar story will describe a D-brane probe that is sent in later: as it approaches the origin of moduli space, it will start to excite W-bosons and other modes, and be trapped by them, by an effect similar to the scattering of D0-branes at small impact parameter [9].

Consider a probe brane at constant $r_{p}$. In the conformally transformed action, the scalar satisfies $\phi=k e^{-\tilde{t} / \ell}$. (This is an exact solution to the DBI action (4.5).) Since the mass of the $\mathrm{W}$-boson is proportional to $\phi$, it will be produced when

$$
\frac{|\dot{\phi}|}{\phi^{2}} \sim 1
$$


This implies that $\phi \sim 1 / \ell$. The bulk radial coordinate is related to $\phi$ by $\tilde{r}=\ell_{s}^{2} \phi \sim \ell_{s}^{2} / \ell$. So the W-bosons are produced well inside the horizon. This is consistent with the fact that infalling observers do not see thermal radiation near the horizon.

If $\mathrm{N}$ is large but finite, the eigenvalues will be trapped for a long time, but eventually come out, and the black hole will eventually decay. We can estimate the time scale over which this occurs, in Schwarzschild coordinates. Consider the $\mu=0$ black hole. The gauge theory on the static spacetime $\Sigma \times \mathbb{R}$ is at finite temperature, $T=\frac{1}{2 \pi \ell}$. The dynamics of a single brane is described by the action (4.3). The static potential starts from $V=0$ at the horizon, rises to a peak, and falls off as $-r^{2}$ at large $r$. We will assume the D-branes start near the horizon; if we form the black hole by a collapsing shell, the D-branes will slow down and strongly fluctuate at this point, so that the state of the dual scalars should have appreciable support near the horizon. For an $M=0$ black hole, the only scale in the integrand of (4.3) is $\ell$, so that the D-brane action scales as:

$$
S=\frac{\ell^{4}}{g_{s}\left(\alpha^{\prime}\right)^{2}} C=N C
$$

where $C$ is a constant of order 1.16 Therefore, we expect that the timescale for emitting a single brane is of order $e^{C N}$, and the time scale for emitting all $N$ branes is of order $N e^{C N}$. This gives a semiclassical process for the black hole to radiate away its charge. The time scale is much longer than the evaporation time for a black hole in asymptotically flat space or for a "small" ten-dimensional black hole in global AdS; on the other hand, it is much shorter than the Poincaré recurrence time for a spherical black hole. The hyperbolic black hole has positive specific heat; without the ability to radiate away D-branes, the black hole would come into thermal equilibrium with its own Hawking radiation and not evaporate, as with planar black holes or large spherical black holes in AdS. The ability to radiate D-branes means that at late time, the black hole will decay (along with the cosmological constant). Since it takes an infinite time for the branes to reach infinity, the asymptotic geometry remains $A d S_{5} \times S^{5}$. The late time behavior is best described by conformally transforming the static cylinder to the expanding cone. In this frame, the scalar eigenvalues are constant (since the outgoing D-branes stay at constant Poincare radius) and the excess energy redshifts to zero.

In previous discussions of the black hole information puzzle, it has often been suggested that violations of locality might occur inside the horizon. In our example, this is clearly

16 For $\mu>0, C$ will be replaced by a function $C\left(r_{+} / \ell\right)$, where $r_{+}$is the horizon radius. 
happening near the singularity. Away from the singularity, local position on a constant- $\tilde{t}$ time slice is well defined since it corresponds to the position of the D-branes or the size of the scalar eigenvalues. However, as we have seen, near the singularity all off-diagonal elements of the scalar matrix become important, all of the eigenvalues interact strongly with these modes and with each other, the D-brane "probes" are no longer good definitions of any geometry, and we expect any notion of geometry to break down. This provides a mechanism for information to come out of the black hole.

What is the best description of the causal structure of the final semi-classical spacetime? It is not just a smoothing out of the spacetime near the singularity with the region outside the horizon unchanged. If that were the case, asymptotic observers who see the shell collapse would not see any branes emerge. The branes would all end up in the second copy of the black hole geometry to the future of the singularity. Since the branes come out in finite time as seen by asymptotic observers, there are two possible Penrose diagrams (see Fig. 4). The figure on the left describes the standard picture of an evaporating black hole in AdS. Since it is clear from the gauge theory that the evolution enters a nongeometric phase (when all the nonabelian degrees of freedom are excited) and there is no loss of unitarity, we think the figure on the right is a more accurate description of the spacetime. The notion of an event horizon clearly requires global causal relations which are not well defined in a spacetime with nongeometric regions. Thus a global event horizon is only well defined in the classical limit and does not have a meaningful quantum analog 17 . More local concepts such as trapped surfaces and apparent horizons will remain meaningful away from the regions of large curvature.

Even though the singularity is resolved in our model, it is not a simple bounce. It takes a long time to pass through the singular region. If a similar picture applied to cosmological singularities, it would have an important implication. It is usually assumed that superhorizon size perturbations propagate unaffected through a bounce. This is because the bounce is assumed to happen quickly at one moment of time, so causality prevents any effects on large scale. This is not the case in our model. Due to the long time delay, there is no causality constraint. Indeed, we expect the W-bosons to eventually decay into inhomogeneous modes and change the spectrum of perturbations.

17 The idea that event horizons may only exist in the classical limit has been discussed before [27] using a different approach to quantum gravity. 

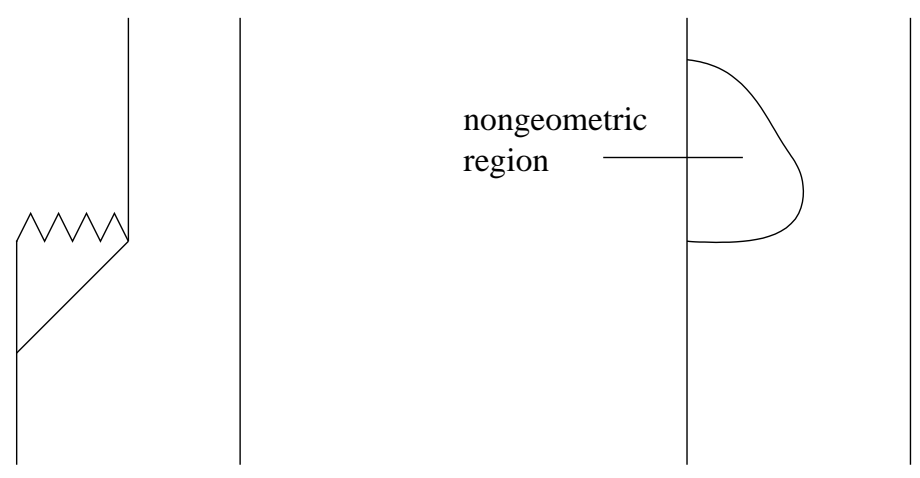

Fig. 4: The diagram on the left is the standard picture of an evaporating black hole in AdS. We believe the diagram on the right is a better description of the physics since it is clear from the gauge theory that the evolution enters a nongeometric phase and remains unitarity.

\section{Generalizations and open questions}

We have focussed on a particularly simple model of a black hole which is locally $A d S_{5}$ and used a "dual" description in terms of Poincare coordinates to show how to describe physics inside the horizon. However the basic mechanism for going inside the horizon is much more general. Consider any black hole in AdS. It can have arbitrary mass, and spherical, planar, or hyperbolic horizon. The usual Schwarzschild coordinates go bad at the horizon since the coordinate $t$ associated with time translations diverges there. One can introduce a good time coordinate associated with constant time surfaces that cross the horizon by a simple shift: $\tilde{t}=t+g(r)$ for some function $g(r)$. To study the motion of an infalling D3-brane one uses the fact that the radial position of the D-brane is associated with the eigenvalue of the super Yang-Mills scalars. The shift to a good time coordinate in the bulk thus corresponds to a field dependent time reparamaterization in the gauge theory. We can also follow D-branes falling into a rotating black hole in AdS. In this case, good coordinates across the horizon are given by $\tilde{\varphi}=\varphi+h(r)$ as well as $\tilde{t}=t+g(r)$ for suitable functions $g, h$. In terms of the dual field theory, both the time and one of the angular variables must undergo field-dependent reparametrizations.

There are many directions for further work. One is to extend our proposal for infalling D-branes to a complete description of the physics inside the horizon in terms of the dual gauge theory. In the simple case of the hyperbolic black hole (with $\mu=0$ ) that we have discussed for most of this paper, this is achieved by going to the Poincare patch description and using the gauge theory on the collapsing cone. But for a general black hole it is not clear what the analogous statement would be. It is possible that this is related to the 
discussion at the end of section 4, concerning the possible connection between our time reparameterization and Yang-Mills gauge transformations.

A second major open question is to gain better control over the singularity. The issue is no longer that our physical theory is breaking down near the singularity; it is clear that evolution in the gauge theory will continue for all time. The point is simply that the dynamics involves all the light degrees of freedom in a complicated way. We believe that our general picture of the singularity will be applicable for all AdS black holes, but the details will differ. For example, when strings propagate on negatively curved spacetimes of decreasing volume, it has been shown that there are many new light degrees of freedom [14], whereas this is not the case for positive curvature. For the hyperbolic black holes, compactifying the hyperbola (as we have done) leads to various complications such as introducing Wilson lines and a nonzero Casimir energy which can break the hyperbolic symmetry. Perhaps a first step toward understanding the physics near the singularity in this case is to consider the uncompactified theory. One question which arises is the following. The breakdown of the DBI action in Schwarzschild coordinates corresponds to thermalization related to the presence of the horizon. The breakdown of the DBI action in coordinates $\tilde{t}, \tilde{r}$ occurs near the singularity. We expect a qualitative difference in the gauge theory between the physics of horizons (thermalization) and that of singularities, since the spacetime near the horizon is weakly curved and classical, while the spacetime near the singularity is strongly curved and the classical approximation is truly breaking down. It would be interesting to find a way to characterize the distinction using our effective actions. 18

A third question is to better understand the $M<0$ black holes from the gauge theory. These have rich causal structures, with multiple asymptotic regions, and inner and outer horizons (which degenerate in the extremal limit). While the singularities appear timelike, the inner horizon is unstable to perturbations which generate initially null singularities that turn over and become spacelike. One possible attack on this class of black holes is to study near-extremal black holes, using deviation from extremality as a small parameter. More general questions are what the collapsing-shell spacetimes look like, and whether there is a presentation of the gauge theory that reaches all of the asymptotic regions as well as the interiors, or whether this is somehow cut off by the instability of the inner horizon. Another interesting regime is for $M$ very small and negative; in this case, while

18 We would like to thank S. Shenker for discussions on this point. 
the gauge theory dynamics should not differ significantly from the $M=0$ case, the causal structure of the black holes at these energies is naively very different.

\section{Acknowledgements}

We are grateful to B. Freivogel, M. Headrick, M. Kleban, J. Maldacena, R. Myers, H. Schnitzer, A. Sever, S. Shenker and S. Trivedi for helpful discussions. Part of this work was done while A.L. and E.S. were attending the workshop "Supersymmetry breaking and its mediation in field theory and string theory" at the Aspen Center for Physics. This work was completed while A.L. and E.S. were attending the "Fundamental Aspects of Superstring Theory" workshop at the Kavli Institute for Theoretical Physics at UC Santa Barbara. E.S. is very grateful to the KITP and the Department of Physics at UCSB for hospitality during several phases of this project. G.H. thanks the Department of Physics at Stanford University for hospitality. A.L. is supported in part by DOE Grant No. DE-FG0292ER40706, and by a DOE Outstanding Junior Investigator award. E.S. is supported by NSF grant PHY-0244728 and by the DOE under contract DE-AC03-76SF00515. A.L. and E.S. are also supported by NSF grant NSF PHY05-51164. G.H. is supported by the NSF grant PHY-0555669. 


\section{References}

[1] P. Kraus, H. Ooguri and S. Shenker, "Inside the horizon with AdS/CFT," Phys. Rev. D 67, 124022 (2003) arXiv:hep-th/0212277; L. Fidkowski, V. Hubeny, M. Kleban and S. Shenker, "The black hole singularity in AdS/CFT," JHEP 0402, 014 (2004) arXiv:hep-th/0306170.

[2] A. Hamilton, D. N. Kabat, G. Lifschytz and D. A. Lowe, "Local bulk operators in AdS/CFT: A holographic description of the black hole interior," Phys. Rev. D 75, 106001 (2007) [Erratum-ibid. D 75, 129902 (2007)] arXiv:hep-th/0612053.

[3] D. Marolf, "Unitarity and Holography in Gravitational Physics," arXiv:0808.2842 [grqc].

[4] R. Emparan, "AdS/CFT duals of topological black holes and the entropy of zeroenergy states," JHEP 9906, 036 (1999) arXiv:hep-th/9906040.

[5] B. Craps, S. Sethi and E. P. Verlinde, "A Matrix Big Bang," JHEP 0510, 005 (2005) arXiv:hep-th/0506180; D. Robbins and S. Sethi, "A matrix model for the nullbrane," JHEP 0602, 052 (2006) arXiv:hep-th/0509204. B. Craps, A. Rajaraman and S. Sethi, "Effective dynamics of the matrix big bang," Phys. Rev. D 73, 106005 (2006) arXiv:hep-th/0601062; E. J. Martinec, D. Robbins and S. Sethi, "Toward the end of time," JHEP 0608, 025 (2006) arXiv:hep-th/0603104.

[6] S. R. Das, J. Michelson, K. Narayan and S. P. Trivedi, Phys. Rev. D 74, 026002 (2006) arXiv:hep-th/0602107]; S. R. Das, J. Michelson, K. Narayan and S. P. Trivedi, "Cosmologies with Null Singularities and their Gauge Theory Duals," Phys. Rev. D 75, 026002 (2007) arXiv:hep-th/0610053; A. Awad, S. R. Das, K. Narayan and S. P. Trivedi, "Gauge Theory Duals of Cosmological Backgrounds and their Energy Momentum Tensors," Phys. Rev. D 77, 046008 (2008) [arXiv:0711.2994 [hep-th]]; A. Awad, S. R. Das, S. Nampuri, K. Narayan and S. P. Trivedi, "Gauge Theories with Time Dependent Couplings and their Cosmological Duals," arXiv:0807.1517 [hep-th].

[7] T. Hertog and G. T. Horowitz, "Towards a big crunch dual," JHEP 0407, 073 (2004) arXiv:hep-th/0406134; T. Hertog and G. T. Horowitz, "Holographic description of AdS cosmologies," JHEP 0504, 005 (2005) arXiv:hep-th/0503071.

[8] B. Craps, T. Hertog and N. Turok, "Quantum Resolution of Cosmological Singularities using AdS/CFT," arXiv:0712.4180 [hep-th].

[9] M. R. Douglas, D. N. Kabat, P. Pouliot and S. H. Shenker, "D-branes and short distances in string theory," Nucl. Phys. B 485, 85 (1997) [arXiv:hep-th/9608024.

[10] E. Silverstein and D. Tong, "Scalar speed limits and cosmology: Acceleration from D-cceleration," Phys. Rev. D 70, 103505 (2004) arXiv:hep-th/0310221.

[11] L. Kofman, A. Linde, X. Liu, A. Maloney, L. McAllister and E. Silverstein, "Beauty is attractive: Moduli trapping at enhanced symmetry points," JHEP 0405, 030 (2004) arXiv:hep-th/0403001. 
[12] C. T. Asplund and D. Berenstein, "Small AdS black holes from SYM," arXiv:0809.0712 [hep-th].

[13] G. R. Dvali, "Infrared hierarchy, thermal brane inflation and superstrings as superheavy dark matter," Phys. Lett. B 459, 489 (1999) arXiv:hep-ph/9905204; J. H. Traschen and R. H. Brandenberger, "Particle production during out-ofequilibrium phase transitions," Phys. Rev. D 42, 2491 (1990).

[14] D. Green, A. Lawrence, J. McGreevy, D. R. Morrison and E. Silverstein, "Dimensional Duality," Phys. Rev. D 76, 066004 (2007) arXiv:0705.0550 [hep-th]].

[15] N. J. Cornish, D. Spergel and G. Starkman, "Can COBE see the shape of the universe?," Phys. Rev. D 57, 5982 (1998) [arXiv:astro-ph/9708225].

[16] D. N. Kabat and G. Lifschytz, "Tachyons and black hole horizons in gauge theory," JHEP 9812, 002 (1998) arXiv:hep-th/9806214; D. N. Kabat and G. Lifschytz, "Gauge theory origins of supergravity causal structure," JHEP 9905, 005 (1999) arXiv:hep-th/9902073; N. Iizuka, D. N. Kabat, G. Lifschytz and D. A. Lowe, "Probing black holes in non-perturbative gauge theory," Phys. Rev. D 65, 024012 (2002) arXiv:hep-th/0108006.

[17] C. V. Johnson, A. W. Peet and J. Polchinski, "Gauge theory and the excision of repulson singularities," Phys. Rev. D 61, 086001 (2000) arXiv:hep-th/9911161.

[18] D. Muller, H. V. Fagundes and R. Opher, "Casimir energy in a small volume multiply connected static hyperbolic pre-inflationary universe," Phys. Rev. D 63, 123508 (2001) arXiv:gr-qc/0103014.

[19] G. T. Horowitz and D. Marolf, "A new approach to string cosmology," JHEP 9807, 014 (1998) arXiv:hep-th/9805207.

[20] V. Balasubramanian, P. Kraus, A. E. Lawrence and S. P. Trivedi, "Holographic probes of anti-de Sitter space-times," Phys. Rev. D 59, 104021 (1999) arXiv:hep-th/9808017.

[21] B. G. Carneiro da Cunha, "Inflation and holography in string theory," Phys. Rev. D 65, 026001 (2002) arXiv:hep-th/0105219.

[22] J. M. Maldacena, "Eternal black holes in Anti-de-Sitter," JHEP 0304, 021 (2003) arXiv:hep-th/0106112.

[23] A. Jevicki, Y. Kazama and T. Yoneya, "Quantum metamorphosis of conformal transformation in D3-brane Yang-Mills theory," Phys. Rev. Lett. 81, 5072 (1998) arXiv:hep-th/9808039.

[24] J. M. Maldacena, "The large N limit of superconformal field theories and supergravity," Adv. Theor. Math. Phys. 2, 231 (1998) [Int. J. Theor. Phys. 38, 1113 (1999)] arXiv:hep-th/9711200.

[25] J. M. Maldacena, "Probing near extremal black holes with D-branes," Phys. Rev. D 57, 3736 (1998) arXiv:hep-th/9705053.

[26] E. Kiritsis, "Supergravity, D-brane probes and thermal super Yang-Mills: A comparison," JHEP 9910, 010 (1999) arXiv:hep-th/9906206.

[27] A. Ashtekar and M. Bojowald, "Black hole evaporation: A paradigm," Class. Quant. Grav. 22, 3349 (2005) arXiv:gr-qc/0504029]. 